\title{
Complementary feeding practices among mothers and nutritional status of infants in Akpabuyo Area, Cross River State Nigeria
}

\author{
Ekerette Emmanuel Udoh ${ }^{*}$ and Olukemi K. Amodu
}

*Correspondence:
ekerette01@gmail.com
Institute of Child Health,
College of Medicine,
University of Ibadan, Ibadan,
Nigeria

*Correspondence: ekerette01@gmail.com College of Medicine, Nigeria

\begin{abstract}
Background: Malnutrition in infants during weaning has been attributed to inappropriate complementary feeding practices and it underlies more than one-third of child mortality in Nigeria. Thus, addressing the influence of complementary feeding practice on nutritional status may be an important approach to reducing the burden of child malnutrition. This cross-sectional study investigated the association between complementary feeding practices among mothers and nutritional status of their infants in Akpabuyo Local Government Area, Nigeria. The study enrolled 330 mother-child pairs from 10 randomly selected out of 32 Health Facilities in Akpabuyo. Socio-demographic information, child and maternal characteristics were obtained using an intervieweradministered questionnaire. Complementary feeding practices were assessed with World Health Organization infant and young child feeding indicators. Nutritional indicators wasting, underweight and stunting were determined.
\end{abstract}

Results: Prevalence of timely introduction of complementary feeding among infants aged $6-8$ months was $85.4 \%$, minimum dietary diversity rate was $31.5 \%$, and minimum meal frequency $36.7 \%$, the rate of minimum acceptable diet was $7.3 \%$. One-third (33.3\%) of the infants were underweight, $26.4 \%$, wasted and 24.6\%, stunted. Children who did not receive timely complementary foods had higher odds for wasting (OR $5.15 ; 95 \% \mathrm{Cl} 1.50-17.73)$. Children who did not receive the minimum dietary diversity had higher odds for underweight than children who received the minimum dietary diversity (OR 2.07; 95\% Cl 1.17-3.70). Children who did not receive the minimum feeding frequency were more likely to be stunted than their peers who received the minimum feeding frequency (OR $1.57 ; 95 \% \mathrm{Cl} 1.53-4.03)$.

Conclusion: Sub-optimal complementary feeding predisposed to infant's malnutrition.

Keywords: Breastfeeding, Complementary feeding, Nutritional status, Infant feeding practise, Bottle feeding, Diarrhoea

\section{Significance}

What is already known? Malnutrition is a significant public health problem in Nigeria. Growth faltering can occur as a result of inadequacy of complementary food in terms of quality, quantity and frequency of meals.

What this study adds? The prevalence of malnutrition in the study area was higher than the national average. Sub-optimal complementary feeding practices among

(c) The Author(s) 2016. This article is distributed under the terms of the Creative Commons Attribution 4.0 International License (http://creativecommons.org/licenses/by/4.0/), which permits unrestricted use, distribution, and reproduction in any medium, provided you give appropriate credit to the original author(s) and the source, provide a link to the Creative Commons license, and indicate if changes were made. 
mothers were risk factors to malnutrition among children in Akpabuyo. Maternal and child's ill health was also significant predictor to child malnutrition.

\section{Background}

Optimal infant feeding practice is an important factor in determining growth and development of a child. For healthy mothers exclusive breast-feeding of infants for up to 6 months is recommended. And at this time when breast milk is no longer sufficient to meet the nutritional requirements of the child especially for energy and micronutrients complementary feeding process is expected to begin. In introducing complementary foods feeding behaviour of mothers change and mothers and/or caregivers adopt various feeding practices which may not comply with standards for optimal infant feeding. Some common practices by mothers during the complementary feeding period bother on the types of food given to infants and the adequacy and frequency of feeding. Food restrictions due to cultural practices, unhygienic practice in bottle feeding, food handling/preparation and responsive breast feeding are also issues of concern during the complementary feeding period for the child (Kruger and Gericke 2003).

Malnutrition in children resulting from inadequate feeding and child care and disease is a major public health problem throughout the developing world including Nigeria (Muller and Krawinkel 2005). Malnutrition is one of the principal underlying causes of death for many of the world's children contributing to more than a third of under-five deaths globally. About 178 million children globally are stunted and Africa has the highest rates (WHO 2012). In Nigeria $41 \%$ of children under- 5 years of age are stunted, with an increase from $27 \%$ at age 6 months to $50 \%$ at 23 months which is the period were complementary feeding intensifies. About $23 \%$ of children under- 5 years are underweight in Nigeria and the prevalence among children aged 6-23 months is 24\%; wasting among under-five children is $13 \%$, and $17 \%$ among children aged 6-23 months. On the other hand obesity stands at $9 \%$ among under-five children (National Population Commision (NPC) and ICF Macro 2009). Generally, the risk of malnutrition in the first 2 years of life has been directly linked with poor breastfeeding and complementary feeding practices of mothers together with high rate of infectious diseases (Arimond and Ruel 2004; Lutter and Rivera 2003; Daelmans and Saadeh 2003). In Nigeria, over 50\% infants are given complementary foods before 6 months and these foods are often of poor nutritional value-mostly inadequate in terms of energy, protein and micronutrients such as iron, zinc, iodine and vitamin A (Federal Ministry of Health [FMH] 2005). Also during the complementary feeding period, the frequency of feeding for the child is usually low, while the quantities and qualities of foods given are usually less than that required for the ages of the child. Thus, complementary feeding process has been associated with major changes in both macronutrient and micronutrient intake of children resulting in malnutrition.

The Millennium Development Goals (MDGs) number 4 targeted the reduction of child mortality by two-third by the year 2015. However, from recent report Nigeria's progress towards reducing child mortality by a two-third since 1990 is off track, with only an average of $1.2 \%$ reduction in under-five mortality yearly (FMH and Save the Children 2011). Annually, nearly one million children die in Nigeria before they reach the age of 
5 years and globally about 11 million under-five children die. In 2006 there was an estimated 9.5 million deaths of children of under- 5 years of age globally and poor nutrition which increases the risk of illness contributed directly or indirectly to more than onethird of these deaths. Undernourished children are regularly prone to measles, malaria, diarrhoea, pneumonia and other illnesses (FMH and Save the Children 2011). Evidence has shown that children who are malnourished and have deficiencies of micronutrient early in life have a lifelong impairment of cognitive and physical development.

Growth faltering as a result of inadequacy of complementary food in terms of quality, quantity and frequency of meals has been reported (Awobgenja and Ugwuona 2010; Kruger and Gericke 2003; Mushaphi et al. 2008). In a study in northern Nigeria on feeding practices and nutritional status of under-five children, Awogbenja and his colleague adduced that mothers who introduced complementary food too early had high incidence of children who were under-nourished (Awogbenja and Ugwuona 2010). Since malnutrition is a significant public health problem in the nation and poor complementary feeding habits of mothers has been linked with poor nutritional status of infants (Awobgenja and Ugwuona 2010; Lutter and Rivera 2003; Arimond and Ruel 2004). Addressing the influence of complementary feeding practice of mothers on nutritional status of children may be an important approach towards reducing the burden of child malnutrition. Therefore, this study describes the complementary feeding options of mothers and its association with the nutritional status of their infants in a rural setting. The findings of the study will assist programme implementers and stakeholders make evidence-based decision in the improvement of children's health by promoting better feeding practices to reduce the prevalence of malnutrition in Nigeria particularly in the vulnerable first year of life.

\section{Methods}

\section{Study setting}

This cross-sectional survey was conducted in Akpabuyo Local Government Area in Cross River State (CRS) Nigeria. Apabuyo has a population of 360,000 people and is located in the Southern Senatorial District with headquarters at Ikot Nakanda (CRS Government 2012). Akpabuyo Local Government Area lies between latitude $4^{\circ} 5^{\prime}$ and $5^{\circ} 40^{\prime}$ and longitude $8^{\circ} 25^{\prime}$ and $8^{\circ} 32^{\prime}$ East. It lies within the vegetation belt of southern Nigeria and shares the Atlantic coastline with Bakassi to the East and the Republic of Cameroon to the West. The Local Government Area measures approximately $28.5 \mathrm{~km}^{2}$ and is predominantly an agricultural area. The major languages spoken are Efik and English, while all the major ethnic groups share a common cultural and ancestral heritage. The people of Akpabuyo are predominantly farmers, traders and fishermen. Data collection for this study was done between April and May which is the beginning of the rainy season and the commencement of a new planting season in the area.

Quantitative data on complementary feeding practices of mothers and nutritional status of their infants were collected using an interviewer administered questionnaire. A total of 10 out of 32 health facilities in the area were randomly selected for use in the study. Sample size calculation was done using Cochran formula (Cochran 1963):

$$
\mathrm{n}=\mathrm{Z}^{2} \mathrm{pq} / \mathrm{d}^{2}
$$


where $\mathrm{n}$ is sample size, $\mathrm{Z}$ is 1.96 (the standard normal deviate), $\mathrm{p}$ equals 0.73 , derived from an estimate of proportions of children age 6-9 months who received complementary food in Nigeria in 2008 (NPC and ICF Macro 2009), $q$ is $1-p$, and ' $d$ ' is the desired level of precision. A $10 \%$ non-response rate and an equal number of participants per health centre were considered; this resulted in a total of 330 mothers with their infants in the age group 6-11 months who were enrolled in this study. A total sampling of 33 eligible mother/child pair from each health facility was done.

The WHO infant and young child feeding questionnaire was used to elicit information on complementary feeding practices (WHO 2010b). A translated version of the pre-tested questionnaire into the local language Efik was carried along with the English version by trained assistants to enable easy communication and recording of data unto the English version. In addition, information on socio-demographic characteristics, maternal and child characteristic were collected. Anthropometric measurement of infants was done to assess nutritional status by measuring weight and height. Weight measurements were done using the Docbel Bruan Baby weighing scale (Docbel Group of Industries, New Delhi, India) and the reading taken to the nearest $0.1 \mathrm{~kg}$. The weighing scale came as a standardized instrument from the manufacturer, however, it was validated by weighing a known weight object to confirm it corresponds with the reading. Height of infants was measured to the nearest $0.1 \mathrm{~cm}$ using a measuring board. The dependent variables weight and recumbent length of the children were used to compute the nutritional status indices; weight-for-age (WFA), weight-for-high (WFH) and Height for age (HFA) expressed in z scores using the 2006 WHO child growth standard reference value (Onis 2007).

\section{Measures}

\section{Complementary feeding indicators}

To assess complementary feeding practices of infants this study adapted the indicators that have been developed due to current recommendations for feeding children according to UNICEF/WHO (WHO 2010a).

\section{Introduction of complementary feeding is the proportion of infants 6-8 months of age who receive solid, semi-solid or soft foods}

Minimum dietary diversity is the proportion of children 6-11 months of age who receive foods from 4 or more food groups. The food group is a score created by adding seven groups of food consumed in the past $24 \mathrm{~h}$. Each food group is given a score of one if food consumed. The food group include; grains, roots, tubers; legumes and nuts; dairy products; flesh foods (meat, fish, poultry, organs; eggs; vitamin A-rich fruits and vegetables, and other fruits and vegetables (WHO 2010a).

Minimum meal frequency implies the proportion of breastfed and non-breastfed children 6-11 months of age who receive solid, semi-solid, or soft foods (also including milk feeds for non-breastfed children) the minimum number of times or more,

Minimum acceptable diet is estimated as the proportion of children 6-11 months of age who receive a minimum acceptable diet (apart from breast milk).

Bottle feeding indicates the proportion of children 6-11 months of age who are fed with a bottle; while; 
Milk feeding frequency for non-breasted child is proportion of non-breastfed children 6-11 months of age who receive at least 2 milk feeding.

\section{Anthropometry}

Weight and height of infants was measured for computing nutritional indicators; [weight-for-height (WFH), weight-for-age (WFA) and height-for-age (HFA) z-scores were determined using WHO anthro v3.2.2. Children were classified as normal (z-score: -2.0 to 2.0 ), wasted (WFH z-score: $<-2.0$ ), underweight (WFA z-score: $<-2.0$ ) and stunted (HFA z-score: <-2.0) (Onis 2007).

\section{Data management and statistical analysis}

Data was entered and analysed using the statistical package IBM SPSS Statistics 20.0. Socio-demographic and economic characteristics, health related characteristics, complementary feeding indicators and nutritional status of infants were first analysed by descriptive statistics. Bivariate analysis was done using Chi square to examine association between independent and dependent categorical variables. Multivariate logistic regression employing forward step-wise method was done to model a relationship between predictor variables and nutritional status indicators. Complementary feeding indicators, socio-demographic/economic, mother's and child's characteristics and household characteristics were combined in a single model. Statistical significance is determined when $\mathrm{p}<0.05$.

\section{Results}

\section{Socio-demographic, maternal and child characteristics}

Table 1 presents the socio-economic characteristics of the respondent mothers. Majority of mothers had a secondary education (48.0\%) and primary education (32.1\%). A third of the mothers were traders (30.6\%) while $27.3 \%$ were either housewife or unemployed; only $0.6 \%$ was professionals or specialist. Most mothers worked outside home (68.8\%) while $31.2 \%$ worked at home. Mothers engaged in daily work exceeding $8 \mathrm{~h}$ was $52.2 \%$ while $46.8 \%$ usually worked for about $4-8 \mathrm{~h}$ daily; $1.1 \%$ were involved in working less than $4 \mathrm{~h}$ daily. Majority of mothers had more than three children (44.3\%). More than a third of the children were reported to have an episode of diarrhoea in the last 2 weeks (39.1\%). Some of the children were reported ill in the previous 1 month preceding the study (48.9\%), as $35.5 \%$ of the mothers also reported to have been ill the 1 month preceding the survey. More of the socio-demographic information of the participants is shown in Table 1.

\section{Complementary feeding practices}

Result showed that only $25.0 \%$ of the mothers started complementary foods at the age of 6 months, while majority had begun complementary feeding before 6 months (73.5\%) and the rest delayed more than 6 months (1.5\%) (See Table 2 on results of complementary feeding outcome). The proportion of infants $6-8$ months of age who were receiving solid, semi-solid or soft foods was $85.4 \%$. The rate of feeding infants the minimum dietary diversity was $31.5 \%$; this rate was lower for infants 6-8 months (25.5\%) than infants aged 9-11 months (43.2\%). Minimum meal frequency rate was $36.7 \%$; higher among children 
Table 1 Socio-demographic, maternal and child characteristics of respondents

\begin{tabular}{|c|c|c|}
\hline Characteristics & $\mathrm{N}$ & $\%$ \\
\hline \multicolumn{3}{|l|}{ Mothers level of education } \\
\hline None & 42 & 12.6 \\
\hline Primary & 107 & 32.1 \\
\hline Secondary & 160 & 48.0 \\
\hline More than secondary & 24 & 7.3 \\
\hline \multicolumn{3}{|l|}{ Mothers occupation } \\
\hline Professional/Specialist & 2 & 0.6 \\
\hline Civil servant & 18 & 5.4 \\
\hline Farmer & 38 & 11.4 \\
\hline Housewife/Unemployed & 91 & 27.3 \\
\hline Student & 46 & 13.8 \\
\hline Trader & 102 & 30.6 \\
\hline Others & 36 & 10.8 \\
\hline \multicolumn{3}{|l|}{ Place of work } \\
\hline Work at home & 59 & 31.2 \\
\hline Work outside home & 130 & 68.8 \\
\hline \multicolumn{3}{|l|}{ Hours of work } \\
\hline$<4$ & 2 & 1.1 \\
\hline $4-8$ & 87 & 46.8 \\
\hline$>8$ & 97 & 52.2 \\
\hline \multicolumn{3}{|l|}{ Mothers income (Naira) } \\
\hline$\leq 10,000$ & 47 & 37.6 \\
\hline $10,001-20,000$ & 43 & 34.4 \\
\hline $20,001-30,000$ & 18 & 14.4 \\
\hline $30,001-40,000$ & 5 & 4.0 \\
\hline $40,001-50,000$ & 7 & 5.6 \\
\hline$>50,000$ & 5 & 4.0 \\
\hline \multicolumn{3}{|c|}{ Number of people in household } \\
\hline$<5$ & 135 & 40.7 \\
\hline $5-9$ & 187 & 56.3 \\
\hline$>9$ & 10 & 3.0 \\
\hline \multicolumn{3}{|l|}{ Parity } \\
\hline 1 & 103 & 31.1 \\
\hline 2 & 82 & 24.7 \\
\hline$\geq 3$ & 147 & 44.3 \\
\hline \multicolumn{3}{|l|}{ Diarrhoea in the last 2 weeks } \\
\hline Yes & 129 & 39.1 \\
\hline No & 201 & 60.9 \\
\hline \multicolumn{3}{|c|}{ Vitamins, minerals, supplements hospital medicine offered in the previous 7 days } \\
\hline Yes & 163 & 49.1 \\
\hline No & 166 & 50.0 \\
\hline Don't know & 3 & 0.9 \\
\hline \multicolumn{3}{|c|}{ Child sick in the previous 1 month } \\
\hline Yes & 161 & 48.9 \\
\hline No & 168 & 51.1 \\
\hline \multicolumn{3}{|c|}{ Child's sickness in the previous 1 month } \\
\hline Fever & 52 & 35.4 \\
\hline Catarrh/cold/cough & 40 & 27.2 \\
\hline
\end{tabular}


Table 1 continued

\begin{tabular}{lcc}
\hline Characteristics & N & $\%$ \\
\hline Malaria & 26 & 17.7 \\
Others & 29 & 19.7 \\
Mother sick in the previous 1 month & & \\
Yes & 116 & 35.5 \\
No & 211 & 64.5 \\
\hline
\end{tabular}

aged 6-8 months (73.4\%) compared with children aged 9-11 months (44.0\%). The rate of minimum acceptable diet was $7.3 \%$, which was lower at age 6-9 months (22.9\%) compared to infants aged 9-11 months (23.4\%). Bottle feeding rate during the previous $24 \mathrm{~h}$ was observed to be $35.1 \%$, higher at age 6-9 months than at age 9-11 months (45.4 vs $16.1 \%)$. Milk feeding frequency for non-breastfed children was $7.0 \%$ and also higher at the age 6-9 months than 9-11 months (7.7 vs 6.7\%).

A-third of the mothers (30.7\%) was of the opinion that their baby was not satisfied with breast milk hence the reason to start complementary food. Some mothers started complementary feeding as a result of instructions given in hospital (18.4\%), while 5.8\% started complementary feeding because breast milk was not enough; $16.4 \%$ reported that baby was always crying as such they began feeding the child with complementary foods. The most common first complementary foods mothers offered to their infants were guinea corn (pap, 37.3\%) and custard (34.2\%). About 20.0\% of mothers gave infant formula alone as the first complementary feed.

Starch based foods were the most consumed food among the children (91.0\%); highest consumption of starchy foods was seen among children aged 9-11 months (95.3\%) and $89.1 \%$ among infants $6-8$ months. Overall dairy product consumption rate was $67.6 \%$ and higher rate, $78.2 \%$, was observed among infants $6-8$ months but $49.2 \%$ among infants aged 9-11 months; consumption of vitamin-A rich fruits and vegetables was $50.8 \%$ and highly consumed among children aged 9-11 months than children 6-8 months (68.9 vs $40.3 \%$ ). Other fruits and vegetable consumption was only $10.8 \%$. Fresh foods consumption was $44.1 \%$, higher among children aged 9-11 months than children $6-8$ months (61.5 vs $34.1 \%)$; consumption of eggs was $9.6 \%$ (13.1\% among children 9-11 months and 7.6\% for children 6-8 months). Legumes and nuts consumption was $18.6 \%$; lower among infants aged 6-8 months than infants 9-11 months of age (16.6 vs $22.1 \%)$.

\section{Nutritional status of infants}

Mean and standard deviation values for the anthropometric indicators WHZ, WAZ and HAZ z-scores were $-0.67 \pm 1.73,-1.10 \pm 1.43,-1.02 \pm 1.25$ respectively. The prevalence of wasting was $26.4 \%$ (95\% CI 21.5-31.3), overweight and obesity was 5.7\% (95\% CI $3.1-8.3$ ), underweight was $33.3 \%$ (95\% CI $28.1-38.5$ ) stunting was $24.6 \%$ (95\% CI $19.8-$ 29.4). Wasting was more common among males compared with females (34.1 vs $18.7 \%$ ) (Table 3), underweight among males was 44.9 and $21.7 \%$ among females. Stunting was $28.7 \%$ for males and $20.5 \%$ among females. Overweight was less common among males 
Table 2 Complementary feeding indicators and related feeding practices of mothers in Apkabuyo

\begin{tabular}{|c|c|c|}
\hline Characteristics & $\mathrm{N}$ & $\%$ \\
\hline \multicolumn{3}{|c|}{ Introduction of solid, semi-solid or soft foods } \\
\hline Met & 135 & 85.4 \\
\hline Not met & 23 & 14.6 \\
\hline \multicolumn{3}{|l|}{ Minimum dietary diversity } \\
\hline Met & 101 & 31.5 \\
\hline Not met & 220 & 68.5 \\
\hline \multicolumn{3}{|l|}{ Minimum meal frequency } \\
\hline Met & 200 & 36.7 \\
\hline Not met & 116 & 63.3 \\
\hline \multicolumn{3}{|l|}{ Minimum acceptable diet } \\
\hline Met & 74 & 23.1 \\
\hline Not met & 247 & 76.9 \\
\hline \multicolumn{3}{|l|}{ Bottle feeding } \\
\hline Yes & 112 & 35.1 \\
\hline No & 207 & 64.9 \\
\hline \multicolumn{3}{|c|}{ Milk feeding frequency for non-breastfed child } \\
\hline Met & 3 & 7.0 \\
\hline Not met & 40 & 93.0 \\
\hline \multicolumn{3}{|l|}{ Still breastfeeding } \\
\hline Yes & 283 & 85.2 \\
\hline No & 49 & 14.8 \\
\hline \multicolumn{3}{|c|}{ Age of introducing complementary food } \\
\hline$<6$ months & 231 & 73.5 \\
\hline 6 months & 86 & 25 \\
\hline$>6$ months & 9 & 1.5 \\
\hline \multicolumn{3}{|c|}{ Separate preparation of child's food } \\
\hline Yes & 225 & 67.6 \\
\hline No & 108 & 32.4 \\
\hline \multicolumn{3}{|c|}{ Reasons for starting complementary foods } \\
\hline Hospital instruction & 62 & 18.4 \\
\hline For good growth & 27 & 8.2 \\
\hline Baby was not satisfied & 101 & 30.7 \\
\hline Breast milk was not enough & 19 & 5.8 \\
\hline Always crying & 54 & 16.4 \\
\hline Hungry & 35 & 10.6 \\
\hline Others & 31 & 9.4 \\
\hline \multicolumn{3}{|c|}{ First complementary food offered to child } \\
\hline Infant formula & 66 & 20.0 \\
\hline Custard & 113 & 34.2 \\
\hline Guinea corn (Pap) & 123 & 37.3 \\
\hline Other foods & 28 & 8.5 \\
\hline \multicolumn{3}{|c|}{ How complementary food is fed to child } \\
\hline Bottle with nipple & 71 & 21.5 \\
\hline Spoon & 222 & 67.3 \\
\hline Cup & 2 & 0.6 \\
\hline Hand & 34 & 10.3 \\
\hline \multicolumn{3}{|c|}{ Hand washing with soap before feeding child } \\
\hline Yes & 173 & 52.3 \\
\hline No & 158 & 47.7 \\
\hline
\end{tabular}


Table 3 Prevalence of malnutrition among infants by sex

\begin{tabular}{llcl}
\hline Indicator & $\begin{array}{l}\text { Males } \\
\%\end{array}$ & $\begin{array}{l}\text { Females } \\
\%\end{array}$ & $\begin{array}{l}\text { All } \\
\% \mathbf{( 9 5 \% ~ C l )}\end{array}$ \\
\hline Wasting (WFH $<-2$ z-score) & 34.1 & 18.7 & $26.4(21.5-31.395 \% \mathrm{Cl})$ \\
Severe wasting (WFH <-3 z-score) & 10.2 & 2.4 & $6.3(3.5-9.195 \% \mathrm{Cl})$ \\
Overweight and obesity (WFH $>+2$ z-score) & 4.2 & 7.2 & $5.7(3.1-8.395 \% \mathrm{Cl})$ \\
Underweight (WFA $<-2$ z-score) & 44.9 & 21.7 & $33.3(28.1-38.595 \% \mathrm{Cl})$ \\
Severe underweight (WFA <-3 z-score) & 12.0 & 2.4 & $7.2(4.9-10.095 \% \mathrm{Cl})$ \\
Stunting (HFA $<-2$ z-score) & 28.7 & 20.5 & $24.6(19.8-29.495 \% \mathrm{Cl})$ \\
Severe stunting (HFA $<-3$ z-score) & 4.8 & 2.4 & $3.6(1.5-5.895 \% \mathrm{Cl})$ \\
\hline
\end{tabular}

compared with females (4.2 vs $7.2 \%$ ). According to age of infants wasting was more prevalent among infants in the age group 9-11 months old (27.0\%) than infants aged 6-8 months (26.3\%), underweight was common among the age group 9-11 months than 6-8 months (34.2 vs 32.9\%), likewise, stunting was more prevalent among 9-11 months old infants than $6-8$ months (34.2 vs $19.7 \%$ ).

\section{Bivariate associations between infant nutritional status and complementary feeding} indicators

Association between infant nutritional status indicators and complementary feeding indicators are presented in Table 4. There was a significant association $(\mathrm{p}<0.05)$ between timely introduction of solids with the nutritional status indicator wasting. Wasting was observed to be more prevalent (39.1\%) among children who did not receive timely solid, semi-solid or soft foods compared with children who received solid, semi-solid or soft foods (24.4\%).

The minimum dietary diversity was significantly associated with underweight. Children who did not receive the minimum dietary diversity were more underweight (38.2\%) than children who received the minimum dietary diversity (23.8\%). Similarly, children who did not receive the minimum acceptable diet were significantly more underweight (36.0.7\%) than their counterparts who received the minimum acceptable diet as recommended (25.7\%).

The feeding indicators introduction of solid, semi-solid or soft foods; minimum dietary diversity and minimum meal frequency showed significant association with stunting. Children who were not receiving solid, semi-solid or soft foods at 6-8 months of age were more stunted than children who received complementary foods at 6-8 months (39.1 vs $20.0 \%$ ); stunting was also more prevalent among infants who were not receiving the minimum dietary diversity $(27.3 \%)$ relative with infants who received the minimum dietary diversity $(16.8 \%)$. Infants who did not receive the minimum meal frequency were more stunted (20.5\%) compared with children who met the minimum feeding frequency criteria.

Wasting in children was higher among infants whose mothers do not wash hands with soap before feeding the child (32.9\%) relative to infants whose mothers washed hands with soap before feeding child (20.8\%) (see Table 4). Infants who had diarrhoea 2 weeks 
Table 4 Wasting, underweight and stunting of infants by complementary feeding indicators, socio-demographic and maternal and child characteristics

\begin{tabular}{|c|c|c|c|c|c|c|c|c|c|}
\hline \multirow[t]{3}{*}{ Complementary feeding indicators } & \multicolumn{9}{|c|}{ Nutritional status of infants $6-11$ months } \\
\hline & \multicolumn{3}{|c|}{ Wasting } & \multicolumn{3}{|c|}{ Underweight } & \multicolumn{3}{|c|}{ Stunting } \\
\hline & $\%$ & Total & $p$ & $\%$ & Total & $p$ & $\%$ & Total & $p$ \\
\hline \multicolumn{10}{|c|}{ Introduction of solid, semi-solid or soft foods } \\
\hline Met & 50.0 & 129 & 0.032 & 32.6 & 135 & 0.835 & 20.0 & 135 & 0.043 \\
\hline Not met & 25.6 & 18 & & 30.4 & 23 & & 39.1 & 23 & \\
\hline \multicolumn{10}{|l|}{ Minimum dietary diversity } \\
\hline Met & 25.0 & 96 & 0.387 & 23.8 & 101 & 0.011 & 16.8 & 101 & 0.042 \\
\hline Not met & 29.8 & 208 & & 38.2 & 220 & & 27.3 & 220 & \\
\hline \multicolumn{10}{|l|}{ Minimum meal frequency } \\
\hline Met & 29.2 & 192 & 0.839 & 34.0 & 200 & 0.931 & 20.5 & 200 & 0.036 \\
\hline Not met & 28.0 & 107 & & 34.5 & 116 & & 31.0 & 116 & \\
\hline \multicolumn{10}{|l|}{ Minimum acceptable diet } \\
\hline Met & 27.1 & 70 & 0.793 & 25.7 & 74 & 0.038 & 14.9 & 74 & 0.036 \\
\hline Not met & 28.8 & 233 & & 36.0 & 247 & & 26.7 & 247 & \\
\hline \multicolumn{10}{|l|}{ Bottle feeding } \\
\hline Yes & 26.5 & 199 & 0.564 & 31.2 & 112 & 0.469 & 25.0 & 112 & 0.791 \\
\hline No & 29.6 & 102 & & 35.2 & 207 & & 23.7 & 207 & \\
\hline \multicolumn{10}{|c|}{ Milk feeding frequency for non-breastfed child } \\
\hline Met & 0.0 & 2 & 0.434 & 0.0 & 3 & 0.356 & 33.3 & 3 & 0.584 \\
\hline Not met & 23.7 & 38 & & 22.5 & 40 & & 20.0 & 40 & \\
\hline \multicolumn{10}{|c|}{ Hand washing with soap before feeding child } \\
\hline Yes & 20.8 & 173 & 0.029 & 28.9 & 173 & 0.062 & 25.4 & 173 & 0.574 \\
\hline No & 32.9 & 158 & & 38.6 & 158 & & 22.8 & 158 & \\
\hline \multicolumn{10}{|l|}{ Diarrhoea in the last 2 weeks } \\
\hline Yes & 36.4 & 129 & 0.016 & 38.0 & 129 & 0.151 & 22.5 & 129 & 0.550 \\
\hline No & 20.4 & 201 & & 30.3 & 201 & & 25.4 & 201 & \\
\hline \multicolumn{10}{|c|}{ Vitamins, minerals, supplements hospital medicine offered in the previous 7 days } \\
\hline Yes & 26.4 & 163 & 0.283 & 35.0 & 163 & 0.640 & 23.3 & 163 & 0.674 \\
\hline No & 26.5 & 166 & & 32.5 & 166 & & 25.3 & 166 & \\
\hline \multicolumn{10}{|l|}{ Child sick in the previous 1 month } \\
\hline Yes & 29.8 & 161 & 0.187 & 41.0 & 161 & 0.004 & 23.6 & 161 & 0.865 \\
\hline No & 23.8 & 168 & & 26.2 & 168 & & 24.4 & 168 & \\
\hline \multicolumn{10}{|l|}{ Mother sick in the previous 1 month } \\
\hline Yes & 19.8 & 116 & 0.060 & 30.2 & 116 & 0.369 & 31.0 & 116 & 0.004 \\
\hline No & 29.9 & 211 & & 35.1 & 211 & & 20.9 & 211 & \\
\hline \multicolumn{10}{|l|}{ Child ever immunised } \\
\hline Yes & 26.3 & 327 & 0.931 & 33.3 & 327 & 1.000 & 24.2 & 327 & 0.670 \\
\hline No & 33.3 & 6 & & 33.3 & 6 & & 16.7 & 6 & \\
\hline \multicolumn{10}{|l|}{ Mother's age } \\
\hline$<20$ & 17.5 & 40 & 0.205 & 25.5 & 40 & 0.067 & 22.5 & 40 & 0.751 \\
\hline $20-30$ & 25.2 & 218 & & 30.7 & 218 & & 22.5 & 218 & \\
\hline$>30$ & 36.5 & 63 & & 44.4 & 63 & & 27.0 & 63 & \\
\hline \multicolumn{10}{|l|}{ Marital status } \\
\hline Single & 16.5 & 19 & 0.000 & 30.4 & 79 & 0.712 & 24.1 & 79 & 0.729 \\
\hline Married & 29.6 & 250 & & 34.4 & 250 & & 24.0 & 250 & \\
\hline Separated & 50.0 & 2 & & 50.0 & 2 & & 0.0 & 2 & \\
\hline \multicolumn{10}{|l|}{ Mother's level of education } \\
\hline None & 31.0 & 42 & 0.750 & 40.5 & 42 & 0.199 & 23.8 & 42 & 0.922 \\
\hline
\end{tabular}


Table 4 continued

\begin{tabular}{|c|c|c|c|c|c|c|c|c|c|}
\hline \multirow[t]{3}{*}{ Complementary feeding indicators } & \multicolumn{9}{|c|}{ Nutritional status of infants $6-11$ months } \\
\hline & \multicolumn{3}{|c|}{ Wasting } & \multicolumn{3}{|c|}{ Underweight } & \multicolumn{3}{|c|}{ Stunting } \\
\hline & $\%$ & Total & $p$ & $\%$ & Total & $p$ & $\%$ & Total & $p$ \\
\hline Primary & 29.0 & 107 & & 38.3 & 107 & & 26.2 & 107 & \\
\hline Secondary & 25.0 & 160 & & 30.0 & 160 & & 22.5 & 160 & \\
\hline More than secondary & 16.7 & 24 & & 20.8 & 24 & & 25.0 & 24 & \\
\hline \multicolumn{10}{|l|}{ Mother's occupation } \\
\hline Professional/Specialist & 0.0 & 2 & 0.000 & 0.0 & 2 & 0.001 & 0.0 & 2 & 0.401 \\
\hline Civil servant & 11.1 & 18 & & 5.6 & 18 & & 5.6 & 18 & \\
\hline Farmer & 44.7 & 38 & & 57.9 & 38 & & 57.9 & 38 & \\
\hline Housewife/Unemployed & 19.8 & 91 & & 29.7 & 91 & & 29.7 & 91 & \\
\hline Student & 21.7 & 48 & & 32.6 & 46 & & 32.6 & 46 & \\
\hline Trader & 33.3 & 102 & & 38.2 & 102 & & 38.2 & 102 & \\
\hline Others & 19.4 & 36 & & 19.4 & 36 & & 19.4 & 36 & \\
\hline \multicolumn{10}{|l|}{ Place of work } \\
\hline Work at home & 39.0 & 59 & 0.272 & 32.2 & 59 & 0466 & 15.3 & 59 & 0.148 \\
\hline Work outside home & 29.2 & 130 & & 37.7 & 130 & & 24.6 & 130 & \\
\hline \multicolumn{10}{|l|}{ Mothers income (Naira) } \\
\hline$\leq 10,000$ & 44.7 & 47 & 0.000 & 48.9 & 47 & 0.008 & 23.4 & 47 & 0.195 \\
\hline $10,001-20,000$ & 32.6 & 43 & & 37.2 & 43 & & 27.9 & 43 & \\
\hline $20,001-30000$ & 5.6 & 18 & & 11.1 & 18 & & 5.6 & 18 & \\
\hline $30,001-40000$ & 20.0 & 5 & & 0.0 & 5 & & 0.0 & 5 & \\
\hline $40,001-50,000$ & 14.3 & 7 & & 14.3 & 7 & & 0.0 & 7 & \\
\hline$>50,000$ & 0.0 & 5 & & 0.0 & 5 & & 20.0 & 5 & \\
\hline \multicolumn{10}{|l|}{ Number of people in household } \\
\hline$<5$ & 26.7 & 135 & 0.447 & 25.9 & 135 & 0.069 & 24.4 & 135 & 0.890 \\
\hline $5-9$ & 26.7 & 187 & & 38.0 & 187 & & 23.2 & 187 & \\
\hline$>9$ & 10.0 & 10 & & 40.0 & 10 & & 24.2 & 10 & \\
\hline \multicolumn{10}{|l|}{ Parity } \\
\hline 1 & 20.0 & 105 & 0.132 & 23.8 & 105 & 0.003 & 19.0 & 105 & 0.219 \\
\hline 2 & 21.7 & 85 & & 27.7 & 83 & & 22.9 & 83 & \\
\hline$\geq 3$ & 33.3 & 144 & & 43.1 & 114 & & 28.5 & 144 & \\
\hline \multicolumn{10}{|l|}{ Child's gender } \\
\hline Male & 34.7 & 167 & 0.006 & 44.3 & 167 & 0.000 & 28.1 & 167 & 0.078 \\
\hline Female & 18.1 & 166 & & 22.3 & 116 & & 19.9 & 144 & \\
\hline \multicolumn{10}{|l|}{ Child's age (months) } \\
\hline $6-8$ & 27.0 & 211 & 0.652 & 32.7 & 211 & 0.826 & 19.4 & 211 & 0.033 \\
\hline $9-11$ & 26.1 & 122 & & 35.1 & 122 & & 32.4 & 122 & \\
\hline \multicolumn{10}{|l|}{ Birth order of child } \\
\hline First & 18.8 & 101 & 0.002 & 23.8 & 101 & 0.005 & 20.8 & 101 & 0.069 \\
\hline Second & 23.1 & 91 & & 30.8 & 91 & & 23.1 & 91 & \\
\hline Third & 18.0 & 50 & & 30.0 & 50 & & 20.0 & 50 & \\
\hline Fourth & 55.6 & 27 & & 55.6 & 27 & & 14.8 & 27 & \\
\hline$>$ Fourth & 37.5 & 64 & & 45.3 & 64 & & 37.5 & 64 & \\
\hline
\end{tabular}

to the study had higher prevalence of wasting (36.4\%) relative to those who did not have diarrhoea (20.4\%). Further analysis showed that of the proportion of infants who were wasted a significant proportion had diarrhoea and their mother did not wash hands with soap before feeding child (50\%) (Table 5). Maternal ill health in the month preceeding 
Table 5 Undernutrition by diarrhoea as function of hand washing with soap before feeding child

\begin{tabular}{|c|c|c|c|c|c|c|c|c|c|c|}
\hline \multirow[t]{2}{*}{ Diarrhoea } & \multirow{2}{*}{$\begin{array}{l}\text { Hand washing } \\
\text { with soap } \\
\text { before feeding } \\
\text { child }\end{array}$} & \multicolumn{3}{|c|}{ Wasting } & \multicolumn{3}{|c|}{ Underweight } & \multicolumn{3}{|c|}{ Stunting } \\
\hline & & $\%$ & Total & $p$ & $\%$ & Total & $p$ & $\%$ & Total & $p$ \\
\hline \multirow[t]{2}{*}{ Yes } & Yes & 25.9 & 58 & 0.006 & 26.2 & 61 & 0.007 & 19.7 & 61 & 0.442 \\
\hline & No & 50.0 & 64 & & 49.3 & 67 & & 25.4 & 67 & \\
\hline \multirow[t]{2}{*}{ No } & Yes & 20.4 & 103 & 0.604 & 30.0 & 110 & 0.865 & 29.1 & 110 & 0.198 \\
\hline & No & 23.5 & 85 & & 31.1 & 90 & & 21.1 & 90 & \\
\hline
\end{tabular}

the survey showed a significant association with child stunting, as $31 \%$ children of these mothers were stunted compared with children whose mothers were not ill (20.9\%).

\section{Multivariate associations for factors predicting infant nutritional status}

In the multivariate association using forward stepwise logistic regression process, selected independent variables were combined in a model with wasting, underweight and stunting to determine factors that remained significant after controlling for confounding effects. The following factors; complementary feeding indicators, socio-demographic/economic, mother's and child's characteristics and household characteristics were combined in a model. All the models were statistically significant, implying that the model with all the factors included significantly improved prediction of the outcome compared with the constant only model. However, the models that presented an excellent fit as the pseudo $\mathrm{R}^{2}$ showed were the adjusted model for underweight and stunting (Tables 6, 7, respectively). The factors that remained significantly associated with wasting in adjusted model were introduction of solid, semi-solid and soft foods and gender of child (see Table 6). Children who did not receive solid, semi-solid and soft foods at 6-8 months were five times more likely to be wasted compared with children who received solid, semi-solid and soft foods (OR 5.15; 95\% CI 1.50-17.73, p < 0.05). Female children were less likely to be wasted compared with their male counterparts (OR 0.18; 95\% CI 0.06-3.59, p < 0.05)

Two factors, minimum dietary diversity and gender of child remained significantly associated with underweight after controlling for maternal, child and household characteristics (Table 7). Children who did not receive the minimum meal diversity were two times more likely to be underweight compared with children who received the minimum meal diversity (OR 2.07; 95\% CI 1.17-3.70, p < 0.05). Female children were less likely be underweight compared with male children (OR 0.34; 95\% CI 0.27-0.61, $\mathrm{p}<0.05$ ).

Infants who did not receive the minimum dietary diversity and minimum meal frequency were significantly more likely to be stunted; (OR 2.17; 95\% CI 1.43-4.20, $\mathrm{p}<0.05)$ and (OR 1.57; 95\% CI 0.53-4.03, $\mathrm{p}<0.05)$ respectively. Maternal illness in the previous 1 month remained significantly associated with stunting (Table 8). Children of mothers who were ill were two times more likely to be stunted compared with children whose mothers were not ill in the previous 1 month (OR 2.35; 95\% CI 1.06-5.22, $\mathrm{p}<0.05$ ) (Table 7) 
Table 6 Adjusted model for factors related with infant wasting

\begin{tabular}{|c|c|c|}
\hline Predictors & Odds ratio $(95 \% \mathrm{Cl})$ & $p$ \\
\hline \multicolumn{3}{|c|}{ Introduction of solid, semi-solid and soft foods } \\
\hline Met (Reference) & 1.00 & \\
\hline Not met & $5.15(1.49-17.73)$ & 0.009 \\
\hline \multicolumn{3}{|l|}{ Marital status } \\
\hline Single (Reference) & 1.00 & \\
\hline Married & $2.55(0.80-7.69)$ & 0.110 \\
\hline \multicolumn{3}{|l|}{ Gender of child } \\
\hline Male (Reference) & 1.00 & \\
\hline Female & $0.18(0.06-0.46)$ & 0.000 \\
\hline \multicolumn{3}{|l|}{ Washing hands with soap } \\
\hline Yes (Reference) & 1.00 & \\
\hline No & $1.56(0.64-3.59)$ & 0.286 \\
\hline \multicolumn{3}{|c|}{ Diarrhoea in the last 2 weeks } \\
\hline No (Reference) & 1.00 & \\
\hline Yes & $1.81(0.76-4.31)$ & 0.180 \\
\hline \multicolumn{3}{|l|}{ Mother's income } \\
\hline$\leq 30,000$ (Reference) & 1.00 & \\
\hline$\geq 30,000$ & $1.06(0.37-3.22)$ & 0.913 \\
\hline \multicolumn{3}{|l|}{ Maternal occupation } \\
\hline Housewife (Reference) & 1.00 & \\
\hline Full/par-time job & $0.59(0.19-1.85)$ & 0.374 \\
\hline
\end{tabular}

Model Chi-Square $=20.562, p=0.021$, Negelkerk $R^{2}=0.423$

\section{Discussion}

In healthy infants introducing complementary feeding must be delayed till 6 months as WHO recommends exclusive breastfeeding for this period to confer several benefits to the infant and mother. Studies have demonstrated that early introduction to solid foods is a risk factor for infection, early cessation of breastfeeding and increased consumption of fatty or sugary foods at 1 year of age (Grummer-Strawn et al. 2008). Our result showed that $2.3 \%$ mothers introduced complementary foods to infants as early as the first month of life and $12.9 \%$ at 2 months. Overall, $73.5 \%$ of the mothers in Akpabuyo introduced complementary food to their infant before the recommended age of 6 months. This high frequency is similar with studies in Nasarawa, Nigeria, where $69.0-82 \%$ mothers are reported to have introduced complementary foods before 6 months (Awogbenja and Ugwuona 2010). In another study in Pakistan, a developing country like Nigeria, lesser prevalence (21\%) of starting complementary foods at an inappropriate time has been recorded (Liaqat et al. 2006). Poor practice of early introduction of complementary foods may be due to the wrong perception mothers have about feeding breast milk alone for the recommended duration of 6 months. In fact we observed that majority of mothers in the study area (30.7\%) believed that the baby was not satisfied with breast milk as such they felt complementary feeding should commence.

Introduction of semi-solid, solid of soft foods at age 6-8 months was higher than the national prevalence of $72.8 \%$ from the 2008 National Demographic Health Survey (NDHS) (NPC and ICF Macro 2009). The high rate of receiving complementary foods at 6-8 months in the present study is encouraging, however, not surprising since a large 
Table 7 Adjusted model for factors related with infant underweight

\begin{tabular}{lll}
\hline Predictors & Odds ratio $(\mathbf{9 5} \% \mathbf{C l})$ & $\boldsymbol{p}$ \\
\hline Minimum dietary diversity & & \\
Met (Reference) & 1.00 & 0.013 \\
Not met & $2.07(1.17-3.70)$ & \\
Gender of child & & 0.000 \\
Male (Reference) & 1.00 & \\
Female & $0.34(0.27-0.61)$ & 0.951 \\
Mother's income & & \\
$\leq 30,000$ (Reference) & 1.00 & 0.262 \\
$\geq 30,000$ & $0.98(0.48-1.96)$ & \\
Maternal occupation & & 0.181 \\
Housewife (Reference) & 1.00 & 0.784 \\
Full/par-time job & $1.47(0.74-2.58)$ & 0.459 \\
Parity & & 0.027 \\
1 (Reference) & 1.00 & 0.557 \\
2 & $0.71(0.07-7.69)$ & 0.685 \\
$\geq 3$ & $2.27(0.25-10.0)$ & 0.844 \\
Birth order of child & & 0.603 \\
First (Reference) & 1.00 & \\
Second & 1.00 & 1.0021 \\
Third & $1.80(1.07-3.01)$ & \\
Fourth & & \\
$>$ Fourth & $1.85(0.18-13.00)$ & \\
Child sick in the previous 1 month & & \\
No (Reference) & & \\
Yes & & \\
\hline
\end{tabular}

Model Chi-Square $=29.822, p=0.001$, Negelkerk $R^{2}=0.678$

Table 8 Adjusted model for factors related with infant stunting

\begin{tabular}{lll}
\hline Predictors & Odds ratio $\mathbf{9 5 \%} \mathbf{C l})$ & $\boldsymbol{p}$ \\
\hline Introduction of solid, semi-solid and soft foods & & 0.499 \\
Met (Reference) & 1.00 & \\
Not met & $1.56(0.42-5.71)$ & 0.043 \\
Minimum dietary diversity & & \\
Met (Reference) & 1.00 & 0.035 \\
Not met & $2.17(1.43-4.20)$ & 0.168 \\
Minimum meal frequency & & \\
Met (Reference) & 1.00 & \\
Not met & $1.57(1.53-4.03)$ & 0.036 \\
Child's age (months) & $0.63(0.33-1.20)$ & \\
Mother sick in the previous 1 month & 1.00 & \\
No (Reference) & $2.35(1.06-5.22)$ & \\
Yes & & \\
\hline Model Chi-Square $=27.655, p=0.011$, Negelkerk $\mathrm{R}^{2}=0.585$ & &
\end{tabular}

percentage of mothers in Akpabuyo begun feeding complementary foods to their infants before the age of 6 months which is inappropriate. The finding of our study is consistent with a study by Marriott et al. (2011) in 14 low-income countries including Nigeria. 
Their study indicated that the rate of infants 6-8 months receiving complementary foods ranged between 50\% in Ethiopia to $90 \%$ in Tanzania. In another study using DHS data and National Family Health Survey data from five South Asian countries, the rate of introduction of solid ranged from 34\% in Pakistan to 84\% in Sri Lanka (Senarath et al. 2012).

The minimum dietary diversity for all infants $6-11$ months was low at $31.5 \%$. Poor diet diversity is a common practice in poor populations because the main complementary diets are mainly starch based staples, with few animal products and vegetables (Arimond and Ruel 2004). Conversely, results indicated the high level at which starchy foods such as grains, roots and tuber foods are being consumed in Akpabuyo compared with more expensive foods like legumes and nuts, eggs and flesh foods which are rich in protein. Many mothers are not buoyant enough to afford expensive animal and vegetable products. Other reasons such as cultural, religious, social, and family influences can limit the types of diets many mothers offer their infants (Odebiyi 1989; Manzoor et al. 2009). Consumption of flesh foods which are rich in iron was encouraging, since iron deficiency is linked with 134,000 deaths annually and regular intake of iron rich foods has been reported to result in the reduction of the prevalence of anaemia among young children from 10 to $40 \%$ in less than a year (World Food Programme and UNICEF 2006).

Dietary diversity in the present study was low. Ajani (2010) reported that in Nigeria dietary diversity is generally poor. In the study which assed dietary diversity in six Nigerian states and employing a total of 14 food group score, $83 \%$ of the children under the age of 5 months received 5-9 food groups. In the present study dietary diversity employed a range of four food groups and above from a score of seven food groups (WHO 2010a). In another study in Zambia and Ethiopia (Disha et al. 2012) which used the current indicator as employed in our study, a low rate for minimum dietary diversity was observed in Ethiopia (7\%) and Zambia (37\%). This data suggest a preponderance of low rate of minimum dietary diversity across low income countries. Minimum feeding frequency was also observed to be low. Low feeding frequency has been attributed to fewer amounts of time mothers put in for care for their infants due to their workload. Many mothers work outside the home and often go out for many hours and sometimes food may not be prepared which can be offered to the child by other family member. Number of meals offered to the child may also depend on the income of the family and who is mainly in control of resources in the family.

The prevalence of minimum acceptable diet for infants aged 6-11 months was low (23.1\%). To meet the minimum acceptable diet mothers need to be able to feed their children diverse diet and the recommended number of meals. This, however, can be difficult for many mothers to achieve in poor societies and who have low economic capacity to secure food for their household.

Although majority of mothers $(64.9 \%)$ did not feed their children with bottle with a nipple, a reasonable proportion (35.1\%) used bottle with a nipple to feed their infants. As a hygiene concern the practice of feeding infants with feeding bottles has been discouraged by WHO because of the difficulties in maintaining feeding bottles that are free of pathogen which can cause infections. Studies have reported high incidence of diarrhoea in the second phase of infancy due to poor hygiene (Bern et al. 1992). A more appropriate means of feeding infants is by the use of spoons or cups. In this study most of the 
mothers $67.2 \%$ fed their infants appropriately using a spoon which is encouraging. The proportion of non-breastfed infants 6-11 months who received at least two milk feedings in the previous day preceding the study was alarmingly low (7\%) considering the fact that these infants are not receiving breast milk. Consumption of diary product generally for all children in this study, however, was seen to be quite high $67.6 \%$.

The overall prevalence of wasting reflecting acute malnutrition among the infants aged 6-11 was very high according to WHO criteria (Onis 2003). However, severe wasting prevalence was low. Overweight and obesity were also low. Underweight which reflects chronic or acute malnutrition among the infants was at a critical level, while severe underweight was low. Stunting which reflects chronic malnutrition was at a medium level while severe stunting was rare. The result indicated that wasting was very high among the males compared with females; underweight prevalence was also very high for both males and females. Prevalence of stunting was at medium level for both males and females. Overweight was less among males compared with females. Higher prevalence of wasting and underweight among males over females have been previously reported (Adeladza 2010; Disha et al. 2012). Results of our study show an apparent increase in malnutrition in infants as children increase in age from 6 months (Marriott et al. 2011; Kumar et al. 2006; NPC and ICF Macro 2009). Wasting increased slightly from $26.3 \%$ among infants aged 6-8 to $27 \%$ among infants 9-11 months. Underweight and stunting also increased with age of the child. As mothers decrease breastfeeding and complementary feeding intensifies, and as the infant ages and the demands for energy and nutrient increase, the quality of complementary foods offered may not be adequate to maintain the better nutritional status the child acquired earlier when breastfeeding was mainly offered with little complementary foods.

Mothers who introduced timely complementary feeding had children with better WHZ than mothers who had not introduced complementary feeding. Similar finding has been observed before in Zambia (Disha et al. 2012). For infants 6-8 months in the Zambia study, consumption of complementary food was positively associated with WHZ score. Result also confirms that introduction of complementary food is significantly associated with stunting after controlling for maternal characteristics, household and child's characteristics. Children who received semi-solid, solid and soft foods at 6-8 months were less stunted (80\%) compared with those who did not receive complementary foods $(60.9 \%)$, this finding is also comparable with another studies (Marriott et al. (2011). The present study indicates that failure in the consumption of complementary foods have the likelihood of resulting in wasting and later stunting of infants: because the indicator introduction of semi-solid, solid or soft food at 6-8 months does not measure the quality or frequency of diets for infants, for wasting and stunting to result due to delay in introduction of complementary food it is obvious that energy need of the young child has not been met.

Our study identified association between underweight and dietary diversity. The association remained after controlling for several factors such as gender of child, mother's income and parity. Children who received the minimum dietary diversity were less likely to be underweight compared with children who did not receive the minimum dietary diversity. Also, children who received the minimum dietary diversity were less likely to be stunted compared with children who did not receive the minimum dietary 
diversity. This observation is in line with previous findings by Marriott et al. (2011). In another study by Arimond and Ruel (2004), the authors demonstrated that children aged 6-23 months who received diverse diet had better HAZ compared with children who did not receive diverse diet.

It was observed that the minimum meal frequency was only associated with stunting but not wasting and underweight and remained significant at multivariate level. Children who received the minimum meal frequency were less stunted compared with children who did not receive the minimum meal frequency. Disha et al. (2012) showed very weak association between feeding frequency and stunting and underweight in Ethiopia. Marriott et al. (2011) in their study, however, found an association between feeding frequency with underweight, but not with stunting. The association between feeding frequency and stunting in the present study is expected as the indicator is mutually inclusive of feeding of complementary food at 6-8 months which equally showed similar association with stunting.

Child Illness still emerged as a predictor of underweight in infants even after controlling for food diversity and gender. This finding support the fact that diseases and infections have direct effect on the nutritional status of children since it affects dietary intake and utilisation. Maternal illness was a significant predictor to child stunting. This probably may be due to reduced capacity to child care, reduced breast feeding and feeding attention that may arise during the period of mother's ill health. In Nigeria, although mothers are the main care providers for children, sometimes mothers ignore their health and their own nutrition, making not only themselves vulnerable to undernutrition, but their children too. There is evidence that adequate maternal knowledge on child feeding is pertinent to better health and development of the child. Although the present study did not investigate the impact of mothers' knowledge on child feeding in the study area and its consequence with children's malnutrition, many mothers in Cross River State lack the knowledge on adequate child feeding (Jemide et al. 2016). It is also important to note that poverty is widespread in many poor societies and the problem of food insecurity as its corollary cannot be overridden as one of the major causes, in addition to lack of maternal knowledge on feeding, of malnutrition among children in the study area.

While this study has provided relevant facts on child malnutrition in the area it was not devoid of some limitations. The limitations included reporting and recall bias a problem common to cross-sectional surveys, particularly for retrospective data relying on memory of past events. This problem in reporting and recall was limited as much as possible by probing respondents to further give details of their responses.

\section{Conclusion}

The prevalence of malnutrition in Akpabuyo based on wasting, underweight and stunting were of public health significance. Children who received timely complementary foods had normal status based on weight-for-height than children who did not receive timely complementary foods even after controlling for maternal, child and health factors. Dietary diversity was a predictor of better weight-for-age. Children who received the minimum feeding frequency had the likelihood of becoming less stunted than their peers who did not receive the minimum feeding frequency. Maternal illness was a significant predictor to child stunting. 


\section{Recommendations}

1. Intervention efforts to improve nutritional status of infants through nutrition and educational inputs should emphasise optimal infants feeding practices.

2. Interventional programmes should target poorer household and mothers with lower educational level to improve complementary feeding practices of mothers.

3. Developmental programmes should focus on empowering women in the community by improving of household income through creation of employment and assess to credit facilities that will enable women engage in sustainable means of livelihood.

4. There is need for support for mothers to commit to continued and adequate care to older children without discrimination to gender to protect children from deteriorating nutritional status as they grow.

5. Caregivers and mothers should be educated on maintenance of hygienic conditions of the home and during feeding of the child to prevent diarrhoea and illness, and on the management of diarrhoea.

6. There is need for promotion of women's health and nutrition as a strategy that will benefit child nutritional status.

\section{Authors' contributions}

EU conceptualised the research and was responsible for data analysis and writing the manuscript. OA checked analysis of the data and interpretation of results, and edited and reviewed the manuscript. Both authors read and approved the final manuscript.

\section{Acknowledgements}

The authors would like to thank Dr. Adesola. O Olumide and Dr. B. A. Ushie for thier contributions in checking the work and providing feedback. The authors also thank the staff of the Institute of Child health, College of Medicine, University of Ibadan for their support and mentorship. We are grateful to Mrs Grace E. Udoh for her passionate support and encouragements during the period of this work. Authors' appreciation also goes to the management of the health facilities in Akpabuyo for granting approval for the study to be carried out in their facilities and also to the participants who gave consent to partake in the research.

\section{Competing interests}

The authors declare that they have no competing interests.

Ethics approval and consent to participate

Ethical approval was obtained from the UCH/UI Ethical Review Committee (No. UI/EC/13/0107). This study only involved individual who showed complete willingness to participate based on attestation to the informed consent provided. Freedom to withdraw from the study at any stage was allowed for the participants.

Received: 11 May 2016 Accepted: 26 November 2016

Published online: 05 December 2016

\section{References}

Adeladza A (2010) The influence of socio-economic and nutritional characteristics on child growth in Kwale District of Kenya. Afr J Food Agric Nutr Dev. doi:10.4314/ajfand.v9i7.47686

Ajani S (2010) An assessment of dietary diversity in six Nigerian States. Afr J Biomed Res 13(3):161-167

Arimond M, Ruel MT (2004) Dietary diversity is associated with child nutritional status: evidence from 11 demographic and health surveys. J Nutr 134(10):2579-2585

Awogbenja MD, Ugwuona FU (2010) Feeding practices and nutritional status of under-five children in Nasarawa State, Nigeria. PAT 6(1):23-35

Bern C, Martines J, De Zoysa I, Glass RI (1992) The magnitude of the global problem of diarrhoeal disease: a ten-year update. Bull World Health Organ 70(6):705

Cochran WG (1963) Sampling Techniques, 2nd edn. John Wiley and Sons Inc, New York

Cross River State (CRS) Government (2012) Akpabuyo Local Government. Retrieved 1st Dec 2012 from www.crossriverstate.gov.ng

Daelmans B, Saadeh R (2003) Global initiatives to improve complementary feeding. SCN News 27:10-18

Disha AD, Rawat R, Subandoro A, Menon P (2012) Infant and young child feeding (IYCF) practices in Ethiopia and Zambia and their association with child nutrition: analysis of demographic and health survey data. Afr J Food Agric Nutr Dev 12(2):5895-5914

Federal Ministry of Health (FMH) (2005) National policy on infant and young child feeding in Nigeria. Abuja, Nigeria 
Federal Ministry of Health (FMH), Save the Children (2011) Saving new-born lives in Nigeria: newborn health in the context of maternal, new-born and child health strategy, 2nd edn. Federal Ministry of Health, Save the Children, Abuja

Grummer-Strawn LM, Scanlon KS, Fein SB (2008) Infant feeding and feeding transitions during the first year of life. Pediatrics. doi:10.1542/peds.2008-1315d

Jemide JO, Ene-Obong HN, Edet EE, Udoh EE (2016) Association of maternal nutrition knowledge and child feeding practices with nutritional status of children in Calabar South Local Government Area, Cross River State. Nigeria. Int J Home Sci 2(1):293-298

Kruger R, Gericke G (2003) A qualitative exploration of rural feeding and weaning practices, knowledge and attitudes on nutrition. Public Health Nutr. doi:10.1079/phn2002419

Kumar D, Goel NK, Mittal PC, Misra P (2006) Influence of infant-feeding practices on nutritional status of under-five children. Indian J Pediatr 73(5):417-421. doi:10.1007/bf02758565

Liaqat P, Rizvi MA, Qayyum A, Ahmed H, Ishtiaq N (2006) Maternal education and complementary feeding. Pak J Nutr 5(6):563-568. doi:10.3923/pjn.2006.563.568

Lutter CK, Rivera JA (2003) Nutritional status of infants and young children and characteristics of their diets. J Nutr 133(9):2941S-2949S

Manzoor I, Daud S, Hashimi NR, Bukhari FSA, Munir MK, Sameer S, Idrees J (2009) Weaning; knowledge and practices. Prof Med J 16(2):279-284

Marriott BP, White A, Hadden L, Davies JC, Wallingford JC (2011) World Health Organization (WHO) infant and young child feeding indicators: associations with growth measures in 14 low-income countries. Maternal Child Nutr 8(3):354370. doi:10.1111/j.1740-8709.2011.00380.x

Muller O, Krawinkel M (2005) Malnutrition and health in developing countries. Can Med Assoc J 173(3):279-286. doi:10.1503/cmaj.050342

Mushaphi L, Mbhenyane X, Khoza L, Amey A (2008) Infant-feeding practices of mothers and the nutritional status of infants in the Vhembe District of Limpopo Province. South Afr J Clin Nutr 21(2):36-41. doi:10.1080/16070658.2008.1 1734159

National Population Commission (NPC) Nigeria, ICF Macro (2009) Nigeria Demographic and Health Survey 2008. National Population Commission and ICF Macro, Abuja

Odebiyi A (1989) Food taboos in maternal and child health: the views of traditional healers in Ile-lfe, Nigeria. Soc Sci Med 28(9):985-996. doi:10.1016/0277-9536(89)90328-6

Onis MD (2003) The World Health Organization Global Database on Child Growth and Malnutrition: methodology and applications. Int J Epidemiol 32(4):518-526. doi:10.1093/ije/dyg099

Onis M (2007) WHO Child Growth Standards based on length/height, weight and age. Acta Paediatr 95:76-85. doi:10.1111/j.1651-2227.2006.tb02378.x

Senarath U, Agho KE, Akram DES, Godakandage SS, Hazir T, Jayawickrama H et al (2012) Comparisons of complementary feeding indicators and associated factors in children aged 6-23 months across five South Asian countries. Maternal Child Nutr 8(s1):89-106

World Food Programme (WFP), UNICEF (2006) Ending childhood hunger and undernutrition initiative: revised global framework for action. UNICEF, New York

World Health Organization (WHO) (2010a) Indicators for assessing infant and young child feeding practices. Part 1, Definitions. WHO, Geneva

World Health Organization (WHO) (2010b) Indicators for assessing infant and young child feeding practices. Part 2, Measure. WHO, Geneva

World Health Organization (WHO) (2012) WHO, nutrition experts take action on malnutrition. Retrieved 19 Dec 2012 from http://www.who.int/nutrition/pressnote_action_on_malnutrition/en/. World Health Organization

\section{Submit your manuscript to a SpringerOpen ${ }^{\circ}$ journal and benefit from:}

- Convenient online submission

- Rigorous peer review

- Immediate publication on acceptance

Open access: articles freely available online

- High visibility within the field

- Retaining the copyright to your article

Submit your next manuscript at $>$ springeropen.com 